Article

\title{
Oscillation Results for Higher Order Differential Equations
}

\author{
Choonkil Park 1,*,+(D), Osama Moaaz ${ }^{2,+}$ (iD) and Omar Bazighifan ${ }^{2,+}$ (D) \\ 1 Research Institute for Natural Sciences, Hanyang University, Seoul 04763, Korea \\ 2 Department of Mathematics, Faculty of Science, Mansoura University, Mansoura 35516, Egypt; \\ o_moaaz@mans.edu.eg (O.M.); o.bazighifan@gmail.com (O.B.) \\ * Correspondence: baak@hanyang.ac.kr \\ + These authors contributed equally to this work.
}

Received: 22 December 2019; Accepted: 25 January 2020; Published: 3 February 2020

\begin{abstract}
The objective of our research was to study asymptotic properties of the class of higher order differential equations with a $p$-Laplacian-like operator. Our results supplement and improve some known results obtained in the literature. An illustrative example is provided.
\end{abstract}

Keywords: ocillation; higher-order; differential equations; $p$-Laplacian equations

\section{Introduction}

In this work, we are concerned with oscillations of higher-order differential equations with a $p$-Laplacian-like operator of the form

$$
\left(r(t)\left|\left(y^{(n-1)}(t)\right)\right|^{p-2} y^{(n-1)}(t)\right)^{\prime}+q(t)|y(\tau(t))|^{p-2} y(\tau(t))=0 .
$$

We assume that $p>1$ is a constant, $r \in C^{1}\left(\left[t_{0}, \infty\right), \mathbb{R}\right), r(t)>0, q, \tau \in C\left(\left[t_{0}, \infty\right), \mathbb{R}\right), q>$ $0, \tau(t) \leq t, \lim _{t \rightarrow \infty} \tau(t)=\infty$ and the condition

$$
\eta\left(t_{0}\right)=\infty
$$

where

$$
\eta(t):=\int_{t}^{\infty} \frac{d s}{r^{1 /(p-1)}(s)} .
$$

By a solution of (1) we mean a function $y \in C^{n-1}\left[T_{y}, \infty\right), T_{y} \geq t_{0}$, which has the property $r(t)\left|\left(y^{(n-1)}(t)\right)\right|^{p-2} y^{(n-1)}(t) \in C^{1}\left[T_{y}, \infty\right)$, and satisfies (1) on $\left[T_{y}, \infty\right)$. We consider only those solutions $y$ of (1) which satisfy $\sup \{|y(t)|: t \geq T\}>0$, for all $T>T_{y}$. A solution of (1) is called oscillatory if it has arbitrarily large number of zeros on $\left[T_{y}, \infty\right)$, and otherwise it is called to be nonoscillatory; (1) is said to be oscillatory if all its solutions are oscillatory.

In recent decades, there has been a lot of research concerning the oscillation of solutions of various classes of differential equations; see [1-24].

It is interesting to study Equation (1) since the $p$-Laplace differential equations have applications in continuum mechanics $[14,25]$. In the following, we briefly review some important oscillation criteria obtained for higher-order equations, which can be seen as a motivation for this paper.

Elabbasy et al. [26] proved that the equation

$$
\left(r(t)\left|\left(y^{(n-1)}(t)\right)\right|^{p-2} y^{(n-1)}(t)\right)^{\prime}+q(t) f(y(\tau(t)))=0,
$$


is oscillatory, under the conditions

$$
\int_{t_{0}}^{\infty} \frac{1}{r^{p-1}(t)} d t=\infty
$$

additionally,

$$
\int_{\ell_{0}}^{\infty}\left(\psi(s)-\frac{1}{p^{p}} \phi^{p}(s) \frac{((n-1) !)^{p-1} \rho(s) a(s)}{\left((p-1) \mu s^{n-1}\right)^{p-1}}-\frac{(p-1) \rho(s)}{a^{1 /(p-1)}(s) \eta^{p}(s)}\right) d s=+\infty,
$$

for some constant $\mu \in(0,1)$ and

$$
\int_{\ell_{0}}^{\infty} k q(s) \frac{\tau(s)^{p-1}}{s^{p-1}} d s=\infty .
$$

Agarwal et al. [2] studied the oscillation of the higher-order nonlinear delay differential equation

$$
\left[\left|y^{(n-1)}(t)\right|^{\alpha-1} y^{(n-1)}(t)\right]^{\prime}+q(t)|y(\tau(t))|^{\alpha-1} y(\tau(t))=0 .
$$

where $\alpha$ is a positive real number. In [27], Zhang et al. studied the asymptotic properties of the solutions of equation

$$
\left[r(t)\left(y^{(n-1)}(t)\right)^{\alpha}\right]^{\prime}+q(t) y^{\beta}(\tau(t))=0, \quad t \geq t_{0} .
$$

where $\alpha$ and $\beta$ are ratios of odd positive integers, $\beta \leq \alpha$ and

$$
\int_{t_{0}}^{\infty} r^{-1 / \alpha}(s) \mathrm{d} s<\infty
$$

In this work, by using the Riccati transformations, the integral averaging technique and comparison principles, we establish a new oscillation criterion for a class of higher-order neutral delay differential Equations (1). This theorem complements and improves results reported in [26]. An illustrative example is provided.

In the sequel, all occurring functional inequalities are assumed to hold eventually; that is, they are satisfied for all $t$ large enough.

\section{Main Results}

In this section, we establish some oscillation criteria for Equation (1). For convenience, we denote that $F_{+}(t):=\max \{0, F(t)\}$,

$$
B(t):=\frac{1}{(n-4) !} \int_{t}^{\infty}(\theta-t)^{n-4}\left(\frac{\int_{\theta}^{\infty} q(s)\left(\frac{\tau(s)}{s}\right)^{p-1} d s}{r(\theta)}\right)^{1 /(p-1)} d \theta
$$

and

$$
D(s):=\frac{r(s) \delta(s)|h(t, s)|^{p}}{p^{p}\left[H(t, s) A(s) \mu \frac{s^{n-2}}{(n-2) !}\right]^{p-1}} .
$$

We begin with the following lemmas.

Lemma 1 (Agarwal [1]). Let $y(t) \in C^{m}\left[t_{0}, \infty\right)$ be of constant sign and $y^{(m)}(t) \neq 0$ on $\left[t_{0}, \infty\right)$ which satisfies $y(t) y^{(m)}(t) \leq 0$. Then,

(I) There exists a $t_{1} \geq t_{0}$ such that the functions $y^{(i)}(t), i=1,2, \ldots, m-1$ are of constant sign on $\left[t_{0}, \infty\right)$; 
(II) There exists a number $k \in\{1,3,5, \ldots, m-1\}$ when $m$ is even, $k \in\{0,2,4, \ldots, m-1\}$ when $m$ is odd, such that, for $t \geq t_{1}$,

$$
y(t) y^{(i)}(t)>0
$$

for all $i=0,1, \ldots, k$ and

$$
(-1)^{m+i+1} y(t) y^{(i)}(t)>0,
$$

for all $i=k+1, \ldots, m$.

Lemma 2 (Kiguradze [15]). If the function $y$ satisfies $y^{(j)}>0$ for all $j=0,1, \ldots, m$, and $y^{(m+1)}<0$, then

$$
\frac{m !}{t^{m}} y(t)-\frac{(m-1) !}{t^{m-1}} y^{\prime}(t) \geq 0
$$

Lemma 3 (Bazighifan [7]). Let $h \in C^{m}\left(\left[t_{0}, \infty\right),(0, \infty)\right)$. Suppose that $h^{(m)}(t)$ is of a fixed sign, on $\left[t_{0}, \infty\right)$, $h^{(m)}(t)$ not identically zero, and that there exists a $t_{1} \geq t_{0}$ such that, for all $t \geq t_{1}$,

$$
h^{(m-1)}(t) h^{(m)}(t) \leq 0 .
$$

If we have $\lim _{t \rightarrow \infty} h(t) \neq 0$, then there exists $t_{\lambda} \geq t_{0}$ such that

$$
h(t) \geq \frac{\lambda}{(m-1) !} t^{m-1}\left|h^{(m-1)}(t)\right|
$$

for every $\lambda \in(0,1)$ and $t \geq t_{\lambda}$.

Lemma 4. Let $n \geq 4$ be even, and assume that $y$ is an eventually positive solution of Equation (1). If (2) holds, then there exists two possible cases for $t \geq t_{1}$, where $t_{1} \geq t_{0}$ is sufficiently large:

$$
\begin{array}{ll}
\left(C_{1}\right) & y^{\prime}(t)>0, y^{\prime \prime}(t)>0, y^{(n-1)}(t)>0, y^{(n)}(t)<0, \\
\left(C_{2}\right) & y^{(j)}(t)>0, y^{(j+1)}(t)<0 \text { for all odd integer } \\
& j \in\{1,2, \ldots, n-3\}, y^{(n-1)}(t)>0, y^{(n)}(t)<0 .
\end{array}
$$

Proof. Let $y$ be an eventually positive solution of Equation (1). By virtue of (1), we get

$$
\left(r(t)\left|\left(y^{(n-1)}(t)\right)\right|^{p-2} y^{(n-1)}(t)\right)^{\prime}<0 .
$$

From ([11] Lemma 4), we have that $y^{(n-1)}(t)>0$ eventually. Then, we can write (4) in the from

$$
\left(r(t)\left(y^{(n-1)}(t)\right)^{p-1}\right)^{\prime}<0
$$

which gives

$$
r^{\prime}(t)\left(y^{(n-1)}(t)\right)^{p-1}+r(t)(p-1)\left(y^{(n-1)}(t)\right)^{p-2} y^{(n)}(t)<0 .
$$

Thus, $y^{(n)}(t)<0$ eventually. Thus, by Lemma 1 , we have two possible cases $\left(C_{1}\right)$ and $\left(C_{2}\right)$. This completes the proof.

Lemma 5. Let y be an eventually positive solution of Equation (1) and assume that Case $\left(C_{1}\right)$ holds. If

$$
\omega(t):=\delta(t)\left(\frac{r(t)\left|\left(y^{(n-1)}(t)\right)\right|^{p-1}}{y^{p-1}(t)}\right)
$$


where $\delta \in C^{1}\left(\left[t_{0}, \infty\right),(0, \infty)\right)$, then

$$
\omega^{\prime}(t) \leq \frac{\delta_{+}^{\prime}(t)}{\delta(t)} \omega(t)-\delta(t) q(t)\left(\frac{\tau^{n-1}(t)}{t^{n-1}}\right)^{p-1}-\frac{(p-1) \mu t^{n-2}}{(n-2) !(\delta(t) r(t))^{1 /(p-1)}} \omega^{p /(p-1)}(t) .
$$

Proof. Let $y$ be an eventually positive solution of Equation (1) and assume that Case $\left(C_{1}\right)$ holds. From the definition of $\omega$, we see that $\omega(t)>0$ for $t \geq t_{1}$, and

$$
\begin{aligned}
\omega^{\prime}(t) \leq & \delta^{\prime}(t) \frac{r(t)\left|\left(y^{(n-1)}(t)\right)\right|^{p-1}}{y^{p-1}(t)}+\delta(t) \frac{\left(r(t)\left|\left(y^{(n-1)}(t)\right)\right|^{p-1}\right)^{\prime}}{y^{p-1}(t)} \\
& -\delta(t) \frac{(p-1) y^{\prime}(t) r(t)\left|\left(y^{(n-1)}(t)\right)\right|^{p-1}}{y^{p}(t)} .
\end{aligned}
$$

Using Lemma 3 with $m=n-1, h(t)=y^{\prime}(t)$, we get

$$
y^{\prime}(t) \geq \frac{\mu}{(n-2) !} t^{n-2} y^{(n-1)}(t)
$$

for every constant $\mu \in(0,1)$. From (5) and (7), we obtain

$$
\begin{aligned}
\omega^{\prime}(t) \leq & \delta^{\prime}(t) \frac{r(t)\left|\left(y^{(n-1)}(t)\right)\right|^{p-1}}{y^{p-1}(t)}+\delta(t) \frac{\left(r(t)\left|\left(y^{(n-1)}(t)\right)\right|^{p-1}\right)^{\prime}}{y^{p-1}(t)} \\
& -\delta(t) \frac{(p-1) \mu t^{n-2}}{(n-2) !} \frac{r(t)\left|\left(y^{(n-1)}(t)\right)\right|^{p}}{y^{p}(t)} .
\end{aligned}
$$

By Lemma 2, we have

$$
\frac{y(t)}{y^{\prime}(t)} \geq \frac{t}{n-1}
$$

Integrating this inequality from $\tau(t)$ to $t$, we obtain

$$
\frac{y(\tau(t))}{y(t)} \geq \frac{\tau^{n-1}(t)}{t^{n-1}}
$$

Combining (1) and (8), we get

$$
\begin{aligned}
\omega^{\prime}(t) \leq & \delta^{\prime}(t) \frac{r(t)\left|\left(y^{(n-1)}(t)\right)\right|^{p-1}}{y^{p-1}(t)}-\delta(t) \frac{q(t)\left(y^{(p-1)}(\tau(t))\right)}{y^{p-1}(t)} \\
& -\delta(t) \frac{(p-1) \mu t^{n-2}}{(n-2) !} \frac{r(t)\left|\left(y^{(n-1)}(t)\right)\right|^{p}}{y^{p}(t)} .
\end{aligned}
$$

From (9) and (10), we obtain

$$
\omega^{\prime}(t) \leq \frac{\delta_{+}^{\prime}(t)}{\delta(t)} \omega(t)-\delta(t) q(t)\left(\frac{\tau^{n-1}(t)}{t^{n-1}}\right)^{p-1}-\frac{(p-1) \mu t^{n-2}}{(n-2) !(\delta(t) r(t))^{1 /(p-1)}} \omega^{p /(p-1)}(t) .
$$

It follows from (11) that

$$
\delta(t) q(t)\left(\frac{\tau^{n-1}(t)}{t^{n-1}}\right)^{p-1} \leq \frac{\delta_{+}^{\prime}(t)}{\delta(t)} \omega(t)-\omega^{\prime}(t)-\frac{(p-1) \mu t^{n-2}}{(n-2) !(\delta(t) r(t))^{1 /(p-1)}} \omega^{p /(p-1)}(t) .
$$

This completes the proof. 
Lemma 6. Let $y$ be an eventually positive solution of Equation (1) and assume that Case $\left(C_{2}\right)$ holds. If

$$
\psi(t):=\sigma(t) \frac{y^{\prime}(t)}{y(t)}
$$

where $\sigma \in C^{1}\left(\left[t_{0}, \infty\right),(0, \infty)\right)$, then

$$
\sigma(t) B(t) \leq-\psi^{\prime}(t)+\frac{\sigma^{\prime}(t)}{\sigma(t)} \psi(t)-\frac{1}{\sigma(t)} \psi^{2}(t) .
$$

Proof. Let $y$ be an eventually positive solution of Equation $(1)$ and assume that Case $\left(C_{2}\right)$ holds. Using Lemma 2, we obtain

$$
y(t) \geq t y^{\prime}(t)
$$

Thus we find that $y / t$ is nonincreasing, and hence

$$
y(\tau(t)) \geq y(t) \frac{\tau(t)}{t}
$$

Since $y>0$, (1) becomes

$$
\left(r(t)\left(y^{(n-1)}(t)\right)^{p-1}\right)^{\prime}+q(t) y^{p-1}(\tau(t))=0 .
$$

Integrating that equation from $t$ to $\infty$, we see that

$$
\lim _{t \rightarrow \infty}\left(r(t)\left(y^{(n-1)}(t)\right)^{p-1}\right)-r(t)\left(y^{(n-1)}(t)\right)^{p-1}+\int_{t}^{\infty} q(s) y^{p-2}(\tau(s))=0 .
$$

Since the function $r\left(y^{(n-1)}\right)^{p-1}$ is positive $\left[r>0\right.$ and $\left.y^{(n-1)}>0\right]$ and nonincreasing $\left(\left(r\left(y^{(n-1)}\right)^{p-1}\right)^{\prime}<0\right)$, there exists a $t_{2} \geq t_{0}$ such that $r\left(y^{(n-1)}\right)^{p-1}$ is bounded above for all $t \geq t_{2}$, and so $\lim _{t \rightarrow \infty}\left(r(t)\left(y^{(n-1)}(t)\right)^{p-1}\right)=c \geq 0$. Then, from (15), we obtain

$$
-r(t)\left(y^{(n-1)}(t)\right)^{p-1}+\int_{t}^{\infty} q(s) y^{p-2}(\tau(s)) \leq-c \leq 0 .
$$

From (14), we obtain

$$
-r(t)\left(y^{(n-1)}(t)\right)^{p-1}+\int_{t}^{\infty} q(s) y(s)^{p-1} \frac{\tau(s)^{p-1}}{s^{p-1}} d s \leq 0
$$

It follows from $y^{\prime}(t)>0$ that

$$
-y^{(n-1)}(t)+\frac{y(t)}{r^{1 /(p-1)}(t)}\left(\int_{t}^{\infty} q(s)\left(\frac{\tau(s)}{s}\right)^{p-1} d s\right)^{1 /(p-1)} \leq 0 .
$$

Integrating the above inequality from $t$ to $\infty$ for a total of $(n-3)$ times, we get

$$
y^{\prime \prime}(t)+\frac{\int_{t}^{\infty}(\theta-t)^{n-4}\left(\frac{\int_{\theta}^{\infty} q(s)\left(\frac{\tau(s)}{s}\right)^{p-1} d s}{r(\theta)}\right)^{1 /(p-1)} d \theta}{(n-4) !} y(t) \leq 0 .
$$


From the definition of $\psi(t)$, we see that $\psi(t)>0$ for $t \geq t_{1}$, and

$$
\psi^{\prime}(t)=\sigma^{\prime}(t) \frac{y^{\prime}(t)}{y(t)}+\sigma(t) \frac{y^{\prime \prime}(t) y(t)-\left(y^{\prime}(t)\right)^{2}}{y^{2}(t)}
$$

It follows from (16) and (17) that

$$
\sigma(t) B(t) \leq-\psi^{\prime}(t)+\frac{\sigma^{\prime}(t)}{\sigma(t)} \psi(t)-\frac{1}{\sigma(t)} \psi^{2}(t) .
$$

This completes the proof.

Definition 1. Let

$$
D=\left\{(t, s) \in \mathbb{R}^{2}: t \geq s \geq t_{0}\right\} \text { and } D_{0}=\left\{(t, s) \in \mathbb{R}^{2}: t>s \geq t_{0}\right\}
$$

We say that a function $H \in C(D, \mathbb{R})$ belongs to the class $\Re$ if

$\left(\mathbf{i}_{1}\right) H(t, t)=0$ for $t \geq t_{0}, H(t, s)>0,(t, s) \in D_{0}$.

$\left(\mathbf{i}_{2}\right) H$ has a nonpositive continuous partial derivative $\partial H / \partial$ s on $D_{0}$ with respect to the second variable.

Theorem 1. Let $n \geq 4$ be even. Assume that there exist functions $H, H_{*} \in \Re, \delta, A, \sigma, A_{*} \in$ $C^{1}\left(\left[t_{0}, \infty\right),(0, \infty)\right)$ and $h, h_{*} \in C\left(D_{0}, \mathbb{R}\right)$ such that

$$
-\frac{\partial}{\partial s}(H(t, s) A(s))=H(t, s) A(s) \frac{\delta^{\prime}(t)}{\delta(t)}+h(t, s) .
$$

and

$$
-\frac{\partial}{\partial s}\left(H_{*}(t, s) A_{*}(s)\right)=H_{*}(t, s) A_{*}(s) \frac{\sigma^{\prime}(t)}{\sigma(t)}+h_{*}(t, s) .
$$

If

$$
\limsup _{t \rightarrow \infty} \frac{1}{H\left(t, t_{0}\right)} \int_{t_{0}}^{t}\left[H(t, s) A(s) \delta(s) q(s)\left(\frac{\tau^{n-1}(s)}{s^{n-1}}\right)^{p-1}-D(s)\right] d s=\infty,
$$

for some constant $\mu \in(0,1)$ and

$$
\limsup _{t \rightarrow \infty} \frac{1}{H_{*}\left(t, t_{0}\right)} \int_{t_{0}}^{t}\left(H_{*}(t, s) A_{*}(s) \sigma(s) B(s)-\frac{\sigma(s)\left|h_{*}(t, s)\right|^{2}}{4 H_{*}(t, s) A_{*}(s)}\right) d s=\infty,
$$

then every solution of (1) is oscillatory.

Proof. Let $y$ be a nonoscillatory solution of Equation (1) on the interval $\left[t_{0}, \infty\right)$. Without loss of generality, we can assume that $y$ is an eventually positive. By Lemma 4, there exist two possible cases for $t \geq t_{1}$, where $t_{1} \geq t_{0}$ is sufficiently large.

Assume that $\left(C_{1}\right)$ holds. From Lemma 5, we get that (6) holds. Multiplying (6) by $H(t, s) A(s)$ and integrating the resulting inequality from $t_{1}$ to $t$, we have

$$
\begin{aligned}
\int_{t_{1}}^{t} H(t, s) A(s) \delta(s) q(s)\left(\frac{\tau^{n-1}(s)}{s^{n-1}}\right)^{p-1} d s \\
\leq-\int_{t_{1}}^{t} H(t, s) A(s) \omega^{\prime}(s) d s+\int_{t_{1}}^{t} H(t, s) A(s) \frac{\delta^{\prime}(s)}{\delta(s)} \omega(s) d s \\
-\int_{t_{1}}^{t} H(t, s) A(s) \frac{(p-1) \mu s^{n-2}}{(n-2) !(\delta(s) r(s))^{1 /(p-1)}} \omega^{p /(p-1)}(s) d s
\end{aligned}
$$


Thus

$$
\begin{gathered}
\int_{t_{1}}^{t} H(t, s) A(s) \delta(s) q(s)\left(\frac{\tau^{n-1}(s)}{s^{n-1}}\right)^{p-1} d s \\
\leq H\left(t, t_{1}\right) A\left(t_{1}\right) \omega\left(t_{1}\right)-\int_{t_{1}}^{t}\left(-\frac{\partial}{\partial s}(H(t, s) A(s))-H(t, s) A(s) \frac{\delta^{\prime}(t)}{\delta(t)}\right) \omega(s) d s \\
-\int_{t_{1}}^{t} H(t, s) A(s) \frac{(p-1) \mu s^{n-2}}{(n-2) !(\delta(s) r(s))^{1 /(p-1)}} \omega^{p /(p-1)}(s) d s
\end{gathered}
$$

This implies

$$
\begin{aligned}
& \int_{t_{1}}^{t} H(t, s) A(s) \delta(s) q(s)\left(\frac{\tau^{n-1}(s)}{s^{n-1}}\right)^{p-1} d s \\
& \leq H\left(t, t_{1}\right) A\left(t_{1}\right) \omega\left(t_{1}\right)+\int_{t_{1}}^{t}|h(t, s)| \omega(s) d(s) \\
&-\int_{t_{1}}^{t} H(t, s) A(s) \frac{(p-1) \mu s^{n-2}}{(n-2) !(\delta(s) r(s))^{1 /(p-1)}} \omega^{p /(p-1)}(s) d s .
\end{aligned}
$$

Using the inequality

$$
\beta U V^{\beta-1}-U^{\beta} \leq(\beta-1) V^{\beta}, \quad \beta>1, U \geq 0 \text { and } V \geq 0
$$

with $\beta=p /(p-1)$,

$$
U=\left((p-1) H(t, s) A(s) \frac{\mu s^{n-2}}{(n-2) !}\right)^{(p-1) / p} \frac{\omega(s)}{(\delta(s) r(s))^{1 / p}}
$$

and

$$
V=\left(\frac{p-1}{p}\right)^{p-1}|h(t, s)|^{p-1}\left(\frac{\delta(s) r(s)}{\left((p-1) H(t, s) A(s) \frac{\mu s^{n-2}}{(n-2) !}\right)^{p-1}}\right)^{(p-1) / p}
$$

we get

$$
\begin{aligned}
|h(t, s)| \omega(s)-H(t, s) A(s) & \frac{(p-1) \mu s^{n-2}}{(n-2) !(\delta(s) r(s))^{1 /(p-1)}} \omega^{p /(p-1)} \\
& \leq \frac{\delta(s) r(s)}{\left(H(t, s) A(s) \frac{\mu s^{n-2}}{(n-2) !}\right)^{p-1}}\left(\frac{|h(t, s)|}{p}\right)^{p}
\end{aligned}
$$

which with (23) gives

$$
\begin{aligned}
\int_{t_{1}}^{t}\left(H(t, s) A(s) \delta(s) q(s)\left(\frac{\tau^{n-1}(s)}{s^{n-1}}\right)^{p-1}-D(s)\right) d s & \leq H\left(t, t_{1}\right) A\left(t_{1}\right) \omega\left(t_{1}\right) \\
& \leq H\left(t, t_{0}\right) A\left(t_{1}\right) \omega\left(t_{1}\right) .
\end{aligned}
$$


Then

$$
\begin{aligned}
\frac{1}{H\left(t, t_{0}\right)} \int_{t_{0}}^{t} & \left(H(t, s) A(s) \delta(s) q(s)\left(\frac{\tau^{n-1}(s)}{s^{n-1}}\right)^{p-1}-D(s)\right) d s \\
& \leq A\left(t_{1}\right) \omega\left(t_{1}\right)+\int_{t_{0}}^{t_{1}} A(s) \delta(s) q(s)\left(\frac{\tau^{n-1}(s)}{s^{n-1}}\right)^{p-1} d s \\
& <\infty,
\end{aligned}
$$

for some $\mu \in(0,1)$, which contradicts (20).

Assume that Case $\left(C_{2}\right)$ holds. From Lemma 6, we get that (13) holds. Multiplying (13) by $H_{*}(t, s) A_{*}(s)$, and integrating the resulting inequality from $t_{1}$ to $t$, we have

$$
\begin{aligned}
\int_{t_{1}}^{t} H_{*}(t, s) A_{*}(s) \sigma(s) B(s) d s \leq & -\int_{t_{1}}^{t} H_{*}(t, s) A_{*}(s) \psi^{\prime}(s) d s+\int_{t_{1}}^{t} H_{*}(t, s) A_{*}(s) \frac{\sigma^{\prime}(s)}{\sigma(s)} \psi(s) d s \\
& -\int_{t_{1}}^{t} \frac{H_{*}(t, s) A_{*}(s)}{\sigma(s)} \psi^{2}(s) d s \\
= & H_{*}\left(t, t_{1}\right) A_{*}\left(t_{1}\right) \psi\left(t_{1}\right)-\int_{t_{1}}^{t} \frac{H_{*}(t, s) A_{*}(s)}{\sigma(s)} \psi^{2}(s) d s \\
& -\int_{t_{1}}^{t}\left(-\frac{\partial}{\partial s}\left(H_{*}(t, s) A_{*}(s)\right)-H_{*}(t, s) A_{*}(s) \frac{\sigma^{\prime}(t)}{\sigma(t)}\right) \psi(s) d s .
\end{aligned}
$$

Then

$$
\begin{aligned}
\int_{t_{1}}^{t} H_{*}(t, s) A_{*}(s) \sigma(s) B(s) d s \leq & H_{*}\left(t, t_{1}\right) A_{*}\left(t_{1}\right) \psi\left(t_{1}\right)+\int_{t_{1}}^{t}\left|h_{*}(t, s)\right| \psi(s) d(s) \\
& -\int_{t_{1}}^{t} \frac{H_{*}(t, s) A_{*}(s)}{\sigma(s)} \psi^{2}(s) d s .
\end{aligned}
$$

Hence we have

$$
\begin{aligned}
\int_{t_{1}}^{t}\left(H_{*}(t, s) A_{*}(s) \sigma(s) B(s)-\frac{\sigma(s)\left|h_{*}(t, s)\right|^{2}}{4 H_{*}(t, s) A_{*}}\right) d s & \leq H_{*}\left(t, t_{1}\right) A_{*}\left(t_{1}\right) \psi\left(t_{1}\right) \\
& \leq H_{*}\left(t, t_{0}\right) A_{*}\left(t_{1}\right) \psi\left(t_{1}\right) .
\end{aligned}
$$

This implies

$$
\begin{aligned}
\frac{1}{H_{*}\left(t, t_{0}\right)} \int_{t_{0}}^{t}\left(H_{*}(t, s) A_{*}\right. & \left.(s) \sigma(s) B(s)-\frac{\sigma(s)\left|h_{*}(t, s)\right|^{2}}{4 H_{*}(t, s) A_{*}}\right) d s \\
& \leq A_{*}\left(t_{1}\right) \psi\left(t_{1}\right)+\int_{t_{0}}^{t} A_{*}(s) \sigma(s) B(s) d s<\infty
\end{aligned}
$$

which contradicts (21). Therefore, every solution of (1) is oscillatory.

In the next theorem, we establish new oscillation results for Equation (1) by using the comparison technique with the first-order differential inequality:

Theorem 2. Let $n \geq 2$ be even and $r^{\prime}(t)>0$. Assume that for some constant $\lambda \in(0,1)$, the differential equation

$$
\varphi^{\prime}(t)+\frac{q(t)}{r(\tau(t))}\left(\frac{\lambda \tau^{n-1}(t)}{(n-1) !}\right)^{p-1} \varphi(\tau(t))=0
$$

is oscillatory. Then every solution of (1) is oscillatory. 
Proof. Let (1) have a nonoscillatory solution $y$. Without loss of generality, we can assume that $y(t)>0$ for $t \geq t_{1}$, where $t_{1} \geq t_{0}$ is sufficiently large. Since $r^{\prime}(t)>0$, we have

$$
y^{\prime}(t)>0, y^{(n-1)}(t)>0 \text { and } y^{(n)}(t)<0 .
$$

From Lemma 3, we get

$$
y(t) \geq \frac{\lambda t^{n-1}}{(n-1) ! r^{1 / p-1}(t)} r^{1 / p-1}(t) y^{(n-1)}(t),
$$

for every $\lambda \in(0,1)$. Thus, if we set

$$
\varphi(t)=r(t)\left[y^{(n-1)}(t)\right]^{p-1}>0,
$$

then we see that $\varphi$ is a positive solution of the inequality

$$
\varphi^{\prime}(t)+\frac{q(t)}{r(\tau(t))}\left(\frac{\lambda \tau^{n-1}(t)}{(n-1) !}\right)^{p-1} \varphi(\tau(t)) \leq 0 .
$$

From [22] (Theorem 1), we conclude that the corresponding Equation (24) also has a positive solution, which is a contradiction.

Theorem 2 is proved.

Corollary 1. Assume that (2) holds and let $n \geq 2$ be even. If

$$
\lim _{t \rightarrow \infty} \inf \int_{\tau(t)}^{t} \frac{q(s)}{r(\tau(s))}\left(\tau^{n-1}(s)\right)^{p-1} d s>\frac{((n-1) !)^{p-1}}{e}
$$

then every solution of (1) is oscillatory.

Next, we give the following example to illustrate our main results.

Example 1. Consider the equation

$$
y^{(4)}(t)+\frac{\gamma}{t^{4}} y\left(\frac{9}{10} t\right)=0, t \geq 1,
$$

where $\gamma>0$ is a constant. We note that $n=4, r(t)=1, p=2, \tau(t)=9 t / 10$ and $q(t)=\gamma / t^{4}$. If we set $H(t, s)=H_{*}(t, s)=(t-s)^{2}, A(s)=A_{*}(s)=1, \delta(s)=t^{3}, \sigma(s)=t, h(t, s)=(t-s)\left(5-3 t s^{-1}\right)$ and $h_{*}(t, s)=(t-s)\left(3-t s^{-1}\right)$ then we get

$$
\eta(s)=\int_{t_{0}}^{\infty} \frac{1}{r^{1 /(p-1)}(s)} d s=\infty
$$

and

$$
\begin{aligned}
B(t) & =\frac{1}{(n-4) !} \int_{t}^{\infty}(\theta-t)^{n-4}\left(\frac{\int_{\theta}^{\infty} q(s)\left(\frac{\tau(s)}{s}\right)^{p-1} d s}{r(\theta)}\right)^{1 /(p-1)} d \theta \\
& =3 \gamma /\left(20 t^{2}\right)
\end{aligned}
$$


Hence conditions (20) and (21) become

$$
\begin{aligned}
& \limsup _{t \rightarrow \infty} \frac{1}{H\left(t, t_{0}\right)} \int_{t_{0}}^{t}\left(H(t, s) A(s) \delta(s) q(s)\left(\frac{\tau^{n-1}(s)}{s^{n-1}}\right)^{p-1}-D(s)\right) d s \\
& \quad=\limsup _{t \rightarrow \infty} \frac{1}{(t-1)^{2}} \int_{1}^{t}\left[\frac{729 \gamma}{1000} t^{2} s^{-1}+\frac{729 \gamma}{1000} s-\frac{729 \gamma}{500} t-\frac{s}{2 \mu}\left(25+9 t^{2} s^{-2}-30 t s^{-1}\right)\right] d s \\
& \quad=\infty(\text { if } \gamma>500 / 81)
\end{aligned}
$$

and

$$
\begin{aligned}
& \limsup _{t \rightarrow \infty} \frac{1}{H_{*}\left(t, t_{0}\right)} \int_{t_{0}}^{t}\left(H_{*}(t, s) A_{*}(s) \sigma(s) B(s)-\frac{\sigma(s)\left|h_{*}(t, s)\right|^{2}}{4 H_{*}(t, s) A_{*}(s)}\right) d s \\
& \quad=\limsup _{t \rightarrow \infty} \frac{1}{(t-1)^{2}} \int_{1}^{t}\left[\frac{3 \gamma}{20} t^{2} s^{-1}+\frac{3 \gamma}{20} s-\frac{3 \gamma}{10} t-\frac{s}{4}\left(9-630 t s^{-1}+t^{2} s^{-2}\right)\right] d s \\
& \quad=\infty(\text { if } \gamma>5 / 3) .
\end{aligned}
$$

Thus, by Theorem 1, every solution of Equation (29) is oscillatory if $\gamma>500 / 81$.

\section{Conclusions}

In this work, we have discussed the oscillation of the higher-order differential equation with a p-Laplacian-like operator and we proved that Equation (1) is oscillatory by using the following methods:

1. The Riccati transformation technique.

2. Comparison principles.

3. The Integral averaging technique.

Additionally, in future work we could try to get some oscillation criteria of Equation (1) under the condition $\int_{t_{0}}^{\infty} \frac{1}{r^{1 /(p-1)}(t)} d t<\infty$. Thus, we would discuss the following two cases:

$$
\begin{array}{ll}
\left(C_{1}\right) & y(t)>0, y^{(n-1)}(t)>0, y^{(n)}(t)<0, \\
\left(C_{2}\right) & y(t)>0, y^{(n-2)}(t)>0, y^{(n-1)}(t)<0 .
\end{array}
$$

Author Contributions: The authors claim to have contributed equally and significantly in this paper. All authors have read and agreed to the published version of the manuscript.

Funding: The authors received no direct funding for this work.

Acknowledgments: The authors thank the reviewers for for their useful comments, which led to the improvement of the content of the paper.

Conflicts of Interest: The authors declare no conflict of interest.

\section{References}

1. Agarwal, R.; Grace, S.; O'Regan, D. Oscillation Theory for Difference and Functional Differential Equations; Kluwer Acad. Publ.: Dordrecht, The Netherlands, 2000.

2. Agarwal, R.; Grace, S.; O'Regan, D. Oscillation criteria for certain nth order differential equations with deviating arguments. J. Math. Appl. Anal. 2001, 262, 601-622. [CrossRef]

3. Agarwal, R.; Shieh, S.L.; Yeh, C.C. Oscillation criteria for second order retarde ddifferential equations. Math. Comput. Model. 1997, 26,1-11. [CrossRef]

4. Agarwal, R.P.; Zhang, C.; Li, T. Some remarks on oscillation of second order neutral differential equations. Appl. Math. Compt. 2016, 274, 178-181. [CrossRef] 
5. Baculikova, B.; Dzurina, J.; Graef, J.R. On the oscillation of higher-order delay differential equations. Math. Slovaca 2012, 187, 387-400. [CrossRef]

6. Bazighifan, O.; Cesarano, C. Some New Oscillation Criteria for Second-Order Neutral Differential Equations with Delayed Arguments. Mathematics 2019, 7, 619. [CrossRef]

7. Bazighifan, O.; Elabbasy, E.M.; Moaaz, O. Oscillation of higher-order differential equations with distributed delay. J. Inequal. Appl. 2019, 55, 1-9. [CrossRef]

8. Chatzarakis, G.E.; Elabbasy, E.M.; Bazighifan, O. An oscillation criterion in 4th-order neutral differential equations with a continuously distributed delay. Adv. Difference Equ. 2019, 336, 1-9.

9. Cesarano, C.; Pinelas, S.; Al-Showaikh, F.; Bazighifan, O. Asymptotic Properties of Solutions of Fourth-Order Delay Differential Equations. Symmetry 2019, 11, 628. [CrossRef]

10. Cesarano, C.; Bazighifan, O. Oscillation of fourth-order functional differential equations with distributed delay. Axioms 2019, 7, 61. [CrossRef]

11. Cesarano, C.; Bazighifan, O. Qualitative behavior of solutions of second order differential equations. Symmetry 2019, 11, 777. [CrossRef]

12. Grace, S.; Dzurina, J.; Jadlovska, I.; Li, T. On the oscillation of fourth order delay differential equations. Adv. Differ. Equ. 2019, 118, 1-15. [CrossRef]

13. Gyori, I.; Ladas, G. Oscillation Theory of Delay Differential Equations with Applications; Clarendon Press: Oxford, UK, 1991.

14. Hale, J.K. Theory of Functional Differential Equations; Springer: New York, NY, USA, 1977.

15. Kiguradze, I.; Chanturia, T. Asymptotic Properties of Solutions of Nonautonomous Ordinary Differential Equations; Kluwer Acad. Publ.: Drodrcht, The Netherlands, 1993.

16. Ladde, G.; Lakshmikantham, V.; Zhang, B. Oscillation Theory of Differential Equations with Deviating Arguments; Marcel Dekker: New York, NY, USA, 1987.

17. Li, T.; Baculikova, B.; Dzurina, J.; Zhang, C. Oscillation of fourth order neutral differential equations with p-Laplacian like operators. Bound. Value Probl. 2014, 56, 41-58. [CrossRef]

18. Moaaz, O. New criteria for oscillation of nonlinear neutral differential equations. Adv. Differ. Equ. 2019, 2019, 484. [CrossRef]

19. Moaaz, O.; Elabbasy, E.M.; Bazighifan, O. On the asymptotic behavior of fourth-order functional differential equations. Adv. Differ. Equ. 2017, 2017, 261. [CrossRef]

20. Moaaz, O.; Elabbasy, E.M.; Muhib, A. Some new oscillation results for fourth-order neutral differential equations. Adv. Differ. Equ. 2019, 2019, 297. [CrossRef]

21. Moaaz, O.; Elabbasy, E.M.; Shaaban, E. Oscillation criteria for a class of third order damped differential equations. Arab J. Math. Sci. 2018, 24, 16-30. [CrossRef]

22. Philos, C. On the existence of nonoscillatory solutions tending to zero at $\infty$ for differential equations with positive delay. Arch. Math. (Basel) 1981, 36, 168-178. [CrossRef]

23. Rehak, P. How the constants in Hille-Nehari theorems depend on time scales. Adv. Differ. Equ. 2006, 2006, 1-15. [CrossRef]

24. Zhang, C.; Agarwal, R.P.; Bohner, M.; Li, T. New results for oscillatory behavior of even-order half-linear delay differential equations. Appl. Math. Lett. 2013, 26, 179-183. [CrossRef]

25. Aronsson, G.; Janfalk, U. On Hele-Shaw flow of power-law fluids. Eur. J. Appl. Math. 1992, 3, $343-366$. [CrossRef]

26. Elabbasy, E.M.; Cesarano, C.; Bazighifan, O.; Moaaz, O. Asymptotic and oscillatory behavior of solutions of a class of higher-order differential equations. Symmetry 2019, 11, 1434. [CrossRef]

27. Zhang, C.; Li, T.; Suna, B.; Thandapani, E. On the oscillation of higher-order half-linear delay differential equations. Appl. Math. Lett. 2011, 24, 1618-1621. [CrossRef]

(C) 2020 by the authors. Licensee MDPI, Basel, Switzerland. This article is an open access article distributed under the terms and conditions of the Creative Commons Attribution (CC BY) license (http:/ / creativecommons.org/licenses/by/4.0/). 